International Journal of Wireless \& Mobile Networks (IJWMN) Vol. 4, No. 5, October 2012

\title{
Parametric Performance Analysis of Patch ANTENNA USING EBG SUBSTRATE
}

\author{
Mst. Nargis Aktar ${ }^{1}$, Muhammad Shahin Uddin ${ }^{1}$, Monir Morshed ${ }^{1}$, \\ Md. Ruhul Amin', and Md. Mortuza $\mathrm{Ali}^{3}$ \\ ${ }^{1}$ Department of Information and Communication Technology \\ Mawlana Bhashani Science and Technology University, Bangladesh \\ ${ }^{2}$ Department of Electrical and Electronic Engineering \\ Islamic University of Technology, Dhaka, Bangladesh \\ ${ }^{3}$ Department of Electrical and Electronic Engineering \\ Rajshahi University of Engineering and Technology, Bangladesh \\ E-mail: \{nargismbstu@gmail.com, shahin.mbstulgmail.com, \\ mm031087@gmail.com, aminr_bd@yahoo.com, and mmali.ruet@gmail.com
}

\begin{abstract}
In recent years, microstrip antennas become very popular because of their interesting advantages of low cost, low profile, light weight, easy fabrication and ease of analysis. Electromagnetic Band Gap (EBG) substrate is used as a part of antenna structure to improve the performance of the patch antenna. Usually, the performance of a patch antenna depends on the parameters such as Return Loss (RL), Bandwidth $(B W)$, Gain, and Directivity. In this paper, we propose a rectangular microstrip patch antenna with EBG substrates of different EBG patch width and analysis the performance of the proposed antenna compare with a conventional patch antenna using the same physical dimension using HFSS simulator. Here we show four analyses of our designed antenna and compare with the conventional microstip patch antenna. Comparison results show that, the performances of our proposed schemes are better comparing the conventional patch antenna.
\end{abstract}

\section{KEYWORDS}

Microstrip Patch Antenna, Electromagnetic Band Gap (EBG) Substrates, Return Loss, Gain, Bandwidth and Directivity, HFSS simulator

\section{INTRODUCTION}

With the drastic demand of wireless communication system and their miniaturization, antenna design becomes more challenging. Recently patch antennas have been widely used. In spite of its several advantages, they suffer from drawbacks such as narrow bandwidth, low gain and excitation of surface waves [1]. To overcome these limitations of microstrip patch antennas two techniques have been used, namely micromachining and periodic structures called the electromagnetic band gap (EBG) structures [13]. However, in recent years there has been considerable effort in the EBG structure for antenna application to suppress the surface wave and overcome the limitations of the antenna. Many works have been done to enhence the performance of the patch antennas [2-11]. The EBG structure utilizes the inherent properties of dielectric materials to enhance the microstrip antenna performance. The characteristics of EBG depend on the shape, size, symmetry and the material used in their construction. There are four main parameters affecting the performance of mushroom like EBG structures such as EBG patch width, gap width, substrates thickness and substrates permittivity [14]. 
International Journal of Wireless \& Mobile Networks (IJWMN) Vol. 4, No. 5, October 2012

In this paper, we propose a rectangular patch antenna with EBG substrates of different EBG patch width, $w$ and investigate the performance of the proposed antenna. The remainder of the paper is organized as follows: In section 2, a brief description of EBG structure. In section 3 present the conventional and proposed antenna design and configuration. In section 4 present the simulation results and discussion. The conclusion of this paper is provided in section 5.

\section{RELATED WORK}

EBG structures are artificially engineered periodic structures with the capability of prohibiting the propagation of electromagnetic waves in a specific frequency band regardless of incident angles and polarization states. They are realized by periodically loading a substrate material with metallic patches [17]. In [2] paper used the mushroom-like EBG structure which was integrated with a patch antenna in various configurations to observe the improvement on the properties of the antenna. Extensive simulation was carried out using CST Microwave Studio. It was observed that with the integration of the EBG structure various improvements were noted in terms of the return loss, directivity, gain and backlobe reduction. In [9] Parametric studies were performed to determine the effect of each parameter on the patch performance, and optimising them for the wide bandwidths and high gainsIn [3], a parametric analysis of the mushroom-type structure and the planar periodic one was presented. The effect of the patch size and the unit cell size on the simultaneous AMC and EBG behavior was discussed, and the influence of the substrate height and the permittivity was studied.

\section{THEORY OF EBG}

Recently there has been growing interest in utilizing electromagnetic band gap (EBG) structures in the electromagnetic and antenna community [7]. EBG structures can be defined as artificial periodic (or sometimes non-periodic) objects that prevent or assist the propagation of electromagnetic waves in a specified band of frequency for all incident angle and polarization state. The main advantage of EBG structure is their ability to suppress the surface wave current that reduce the antenna efficiency and radiation pattern. In mushroom like EBG structure, a band gap is observed between the frequency $7 \mathrm{GHz}$ and $11 \mathrm{GHz}$. On the other hand, for the uniplanar EBG surfaces a band gap is observed the frequency from $13 \mathrm{GHz}$ to $14.6 \mathrm{GHz}$. In this paper, mushroom like EBG surface is used in order to design patch antenna on EBG substrates because it has a lower frequency band gap and a wider bandwidth than the uniplanar EBG surface. A two dimensional mushroom like EBG structure is shown in Figure 3.1. Design of patch antenna mushroom like EBG structures are preferable because light weight, low fabrication cost. The parametric study of mushroom like EBG structure is presented in [12-13]. The affecting parameters for the performance of the proposed antenna are EBG patch width $w$, gap width $g$, substrates thickness $\mathrm{h}$ and substrates permittivity $\varepsilon_{r}$ that are directly depend on the operating wavelength of the patch antenna $[8,18]$.The parameters are varying with operating wavelength as like this that the EBG patch width varies from $0.04 \lambda_{12 \mathrm{GHz}}$ to $0.20 \lambda_{12 \mathrm{GHz}}$, gap width varies from $0.01 \lambda_{12 \mathrm{GHz}}$ to $0.12 \lambda_{12 \mathrm{GHz}}$ and the substrate thickness varies from $0.01 \lambda_{12 \mathrm{GHz}}$ to $0.09 \lambda_{12 \mathrm{GHz}}$. Here, $\lambda_{12}$ means the wavelength between medium 1 and 2 i.e. the free space and the guiding device and $\mathrm{GHz}$ means the wavelength respect to the $\mathrm{GHz}$ range frequency [14]. In this paper, mushroom like EBG structure is integrated with the proposed antenna, the study is focusing with different EBG patch width and the other parameters of the EBG structure is consider constant and analysis the performance parameter of the proposed antenna at different EBG patch width. 

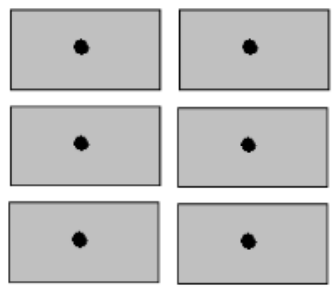

(a) Top view

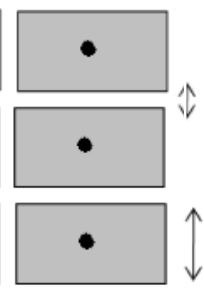

width, w

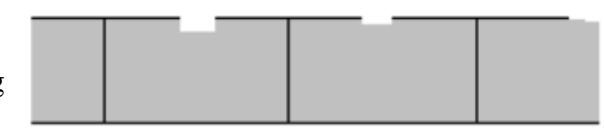

(b) Cross view

Figure 3.1 Two dimensional mushrooms like EBG surfaces: (a) Top view (b) Cross view

\section{Antenna Design ANd Configuration}

In order to identify and verify the enhancement of the performance of patch antenna on EBG substrates, designed a conventional antenna and the proposed antennas. The width, $W$ of the rectangular patch antenna is usually chosen to be larger than the length of the patch, $L$ to get higher bandwidth. The resonant frequency of the antenna is $10 \mathrm{GHz}$. In this paper, we use Neltec dielectric material as patch substrates whose dielectric constant is 2.45 that is low dielectric constant, in low dielectric constant surface wave losses are more severe and dielectric and conductor losses are less severe. By using EBG substrates, surface wave loss can be reduced easily. The antenna is excited by a microstrip transmission line feed. The point of excitation is adjustable to control the impedance match between feed and antenna, polarization, mode of operation and excitation frequency [15-16]. Table1 shows the important parameters for the geometrical configuration of the patch antenna.

Table1 Geometrical configuration of the patch antenna

\begin{tabular}{|l|l|l|}
\hline Antenna Part & Parameter & Value \\
\hline \multirow{2}{*}{ Patch } & Length & $8.8 \mathrm{~mm}$ \\
\cline { 2 - 3 } & Width & $11.4 \mathrm{~mm}$ \\
\hline \multirow{2}{*}{$\begin{array}{l}\text { Patch Substrates } \\
\text { (NeltecN×9245) } \\
(\mathrm{IM})(\mathrm{tm})\end{array}$} & Dielectric constant & 2.45 \\
\cline { 2 - 3 } & Height & $0.787 \mathrm{~mm}$ \\
\cline { 2 - 3 } & Dielectric loss tangent & 0.01 \\
\hline \multirow{3}{*}{ EBG Substrates } & EBG patch Width & $(0.08$ to 0.20$) \lambda_{12} \mathrm{GHz}$ \\
\cline { 2 - 3 } & Gap Width & $0.02 \lambda_{12} \mathrm{GHz}$ \\
\cline { 2 - 3 } & Substrates thickness & $0.04 \lambda_{12 \mathrm{GHz}}$ \\
\hline Operating Frequency & & $10 \mathrm{GHz}$ \\
\hline
\end{tabular}

In this paper, to analysis the performance parameter of the proposed antenna at different EBG patch width $w$, we have designed five antennas integrated with different EBG patch width and defined their name as Antenna-1, Antenna-2, Antenna-3, Antenna-4, Antenna-5 that are given below 
Antenna-1:

In case of Antenna-1, EBG patch width, $w=0.08 \lambda_{12 \mathrm{GHz}}$, gap width, $g=0.02 \lambda_{12 \mathrm{GHz}}$, substrates thickness, $h=0.04 \lambda_{12} \mathrm{GHz}$

Antenna-2:

In case of Antenna-2, EBG patch width, $w=0.10 \lambda_{12 \mathrm{GHz}}$, gap width, $g=0.02 \lambda_{12 \mathrm{GHz}}$, substrates thickness, $h=0.04 \lambda_{12} \mathrm{GHz}$

Antenna-3:

In case of Antenna-3, EBG patch width, $w=0.12 \lambda_{12 \mathrm{GHz}}$, gap width, $g=0.02 \lambda_{12 \mathrm{GHz}}$, substrates thickness, $h=0.04 \lambda_{12 \mathrm{GHz}}$

Antenna-4:

In case of Antenna-4, EBG patch width, $w=0.16 \lambda_{12 \mathrm{GHz}}$, gap width, $g=0.02 \lambda_{12 \mathrm{GHz}}$, substrates thickness, $h=0.04 \lambda_{12 \mathrm{GHz}}$

Antenna-5:

In case of Antenna-5, EBG patch width, $w=0.20 \lambda_{12 \mathrm{GHz}}$, gap width, $g=0.02 \lambda_{12 \mathrm{GHz}}$, substrates thickness, $h=0.04 \lambda_{12 \mathrm{GHz}}$

\section{SimUlation RESUlTS AND DISCUSSION}

Now-a-days, it is a common practice to evaluate the system performances through computer simulation before the real time implementation. A simulator "Ansoft HFSS" based on finiteelement method (FEM) has been used to calculate return loss, impedance bandwidth, radiation pattern and gains. This simulator also helps to reduce the fabrication cost because only the antenna with the best performance would be fabricated.

\subsection{RETURN LOSS}

The simulated return losses that are obtained from conventional antenna and the antennas using EBG substrates are given in Table 5.1. From the table, it is seen that the return loss of the antenna with EBG structure is less compared to the conventional antenna. It is also seen that when the EBG patch width increases than the return loss also increases. Therefore, the antenna performance is better than the conventional antenna because the return loss is reduced for the EBG structure.

Table 2 Return loss of conventional antenna and the proposed antennas using EBG substrates

\begin{tabular}{|l|c|c|}
\hline \multicolumn{1}{|c|}{ Antenna Name } & EBG Patch width & Return Loss in dB \\
\hline Conventional Antenna & & -14.5 \\
\hline Antenna-1 & $0.08 \lambda_{12} \mathrm{GHZ}$ & -23 \\
\hline Antenna-2 & $0.10 \lambda_{12} \mathrm{GHZ}$ & -22.8 \\
\hline Antenna-3 & $0.12 \lambda_{12 \mathrm{GHZ}}$ & -21 \\
\hline Antenna-4 & $0.16 \lambda_{12 \mathrm{GHZ}}$ & -20 \\
\hline Antenna-5 & $0.20 \lambda_{12 \mathrm{GHZ}}$ & -19.3 \\
\hline
\end{tabular}

The return loss versus EBG patch width of the proposed antenna is shown in Figure 5.1 
International Journal of Wireless \& Mobile Networks (IJWMN) Vol. 4, No. 5, October 2012

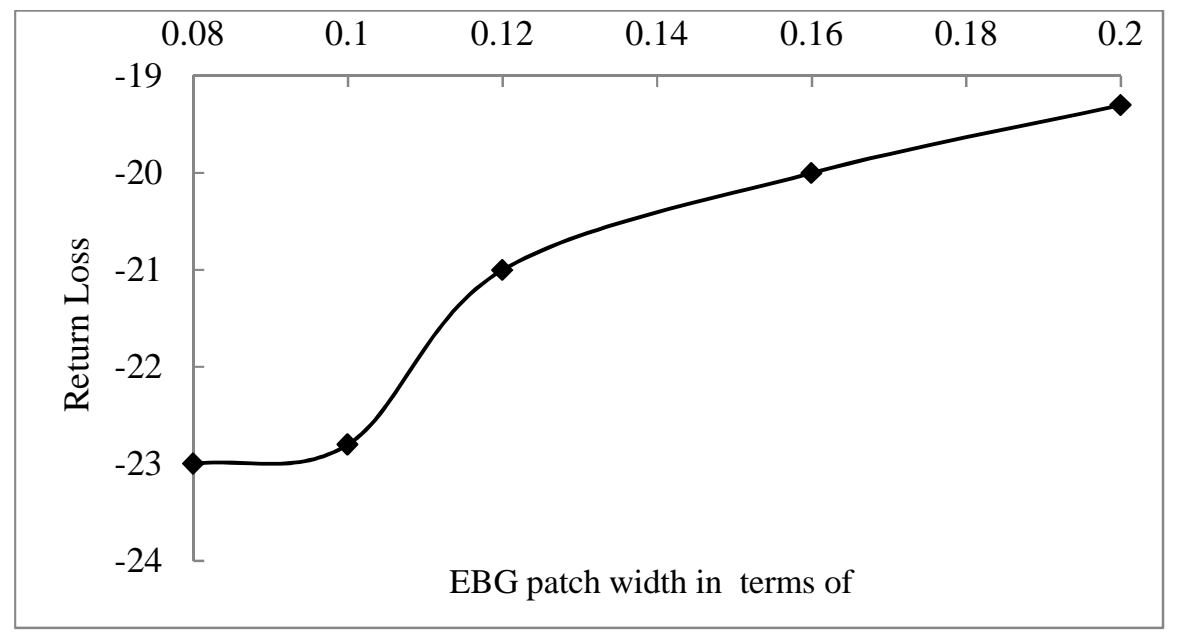

Fig 5.1 Return loss versus EBG patch width of the proposed antenna

Figure 5.1 shows the simulated results of the return loss of a patch antenna on EBG structures with different EBG patch width. Here the return loss is increasing when the EBG patch width is increasing.

\subsection{BANDWIDTH (BW)}

The simulated bandwidth that are obtained from the conventional antenna and the antennas integrated with EBG structures are given in Table 3

Table 3 Bandwidth of conventional antenna and the antennas on EBG substrates

\begin{tabular}{|c|c|c|}
\hline Antenna Name & EBG patch width & Bandwidth in $\mathrm{MHz}$ \\
\hline Conventional Antenna & & 240 \\
\hline Antenna-1 & $0.08 \lambda_{12} \mathrm{GHZ}$ & 330 \\
\hline Antenna-2 & $0.10 \lambda_{12 \mathrm{GHZ}}$ & 330 \\
\hline Antenna-3 & $0.12 \lambda_{12 \mathrm{GHZ}}$ & 320 \\
\hline Antenna-4 & $0.16 \lambda_{12 \mathrm{GHZ}}$ & 300 \\
\hline Antenna-5 & $0.20 \lambda_{12} \mathrm{GHz}$ & 280 \\
\hline
\end{tabular}

From the table 3, it is seen that the bandwidth of the antenna with EBG structure is higher than the conventional antenna. Therefore, the performance of the antenna with EBG structure is better than the conventional antenna because the bandwidth is increased for the EBG structure. The bandwidth versus EBG patch width of the antenna using EBG substrates is shown in the Figure 5.2

From the Figure 5.2, it is seen that, firstly bandwidth is constant but when increase the EBG patch width then decrease the bandwidth. 
International Journal of Wireless \& Mobile Networks (IJWMN) Vol. 4, No. 5, October 2012

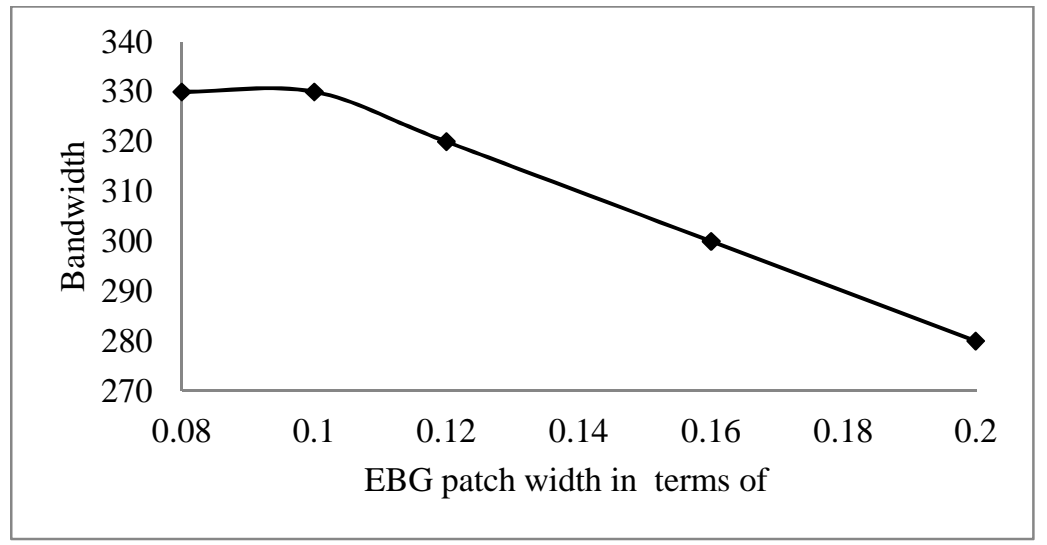

Figure 5.2 Bandwidth versus EBG patch width of the antenna on EBG substrates

\subsection{GAIN}

The simulated gains that are obtained from conventional antenna and the antennas using EBG substrates are in Table 4

Table 4 Gain of conventional antenna and the proposed antennas on EBG substrates

\begin{tabular}{|l|c|c|}
\hline \multicolumn{1}{|c|}{ Antenna Name } & EBG patch width & Gain in $\mathrm{dBi}$ \\
\hline Conventional Antenna & & 22.3 \\
\hline Antenna-1 & $0.08 \lambda_{12 \mathrm{GHZ}}$ & 24.6 \\
\hline Antenna-2 & $0.10 \lambda_{12 \mathrm{GHZ}}$ & 25.7 \\
\hline Antenna-3 & $0.12 \lambda_{12 \mathrm{GHZ}}$ & 25.7 \\
\hline Antenna-4 & $0.16 \lambda_{12 \mathrm{GHZ}}$ & 24.4 \\
\hline Antenna-5 & $0.20 \lambda_{12 \mathrm{GHZ}}$ & 24.2 \\
\hline
\end{tabular}

Table 4 shows the simulation results of the gain of conventional antenna and the antennas on EBG substrates with different EBG patch width. The table shows that the gain of the proposed antenna is higher than the conventional antenna.

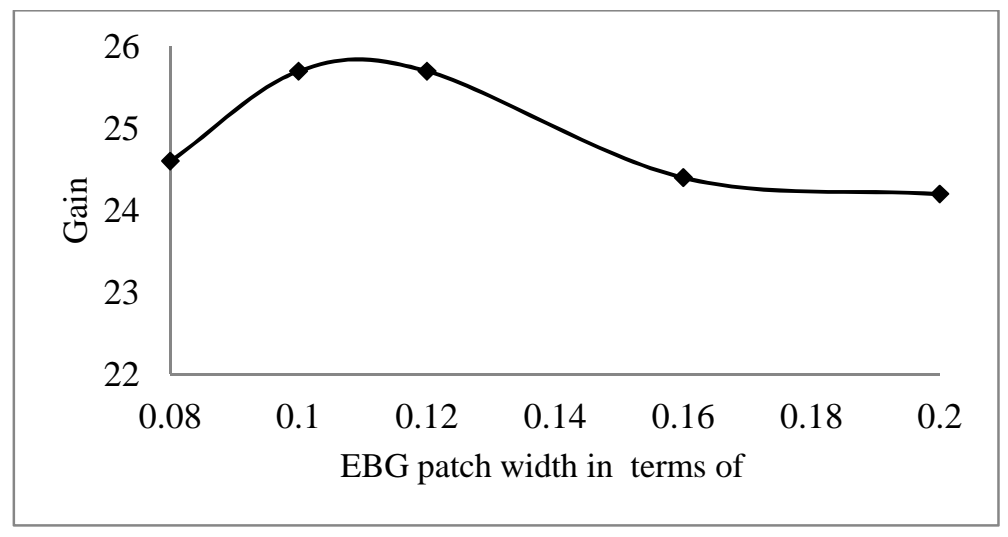

Figure 5.3 Gain versus EBG patch width of the antenna on EBG substrates 
The gain versus EBG patch width of the proposed antenna is shown in the Figure 5.3. Here shows the simulated results of the gain variation of a patch antenna on EBG structures with different EBG patch width. The Figure shows that the gain is increasing with the EBG patch width from 0.08 to 0.1 and then the middle part the gain is almost constant. After that EBG patch width from 0.12, the gain is decreasing in nature with the increasing EBG patch width.

\subsection{DIRECTIVITY}

The simulated directivity that are obtained from conventional antenna and the antennas on EBG substrates are given in Table 5

Table 5 below shows the simulation results of the directivity of the conventional antenna and the antenna on EBG substrates with different EBG patch width. From the table, it is seen that the directivity of the antenna with EBG structure is higher than the conventional antenna.

Table 5 Directivity of conventional antenna and the antennas on EBG substrates

\begin{tabular}{|l|c|c|}
\hline \multicolumn{1}{|c|}{ Antenna Name } & EBG patch width & Directivity in $\mathrm{dB}$ \\
\hline Conventional Antenna & & 23.4 \\
\hline Antenna-1 & $0.08 \lambda_{12} \mathrm{GHZ}$ & 25.5 \\
\hline Antenna-2 & $0.10 \lambda_{12 \mathrm{GHZ}}$ & 25.6 \\
\hline Antenna-3 & $0.12 \lambda_{12 \mathrm{GHZ}}$ & 25.6 \\
\hline Antenna-4 & $0.16 \lambda_{12 \mathrm{GHZ}}$ & 24.3 \\
\hline Antenna-5 & $0.20 \lambda_{12} \mathrm{GHZ}$ & 24.1 \\
\hline
\end{tabular}

Figure 5.4 shows the simulated results of the directivity variations of a patch antenna on EBG structures with different EBG patch width. The Figure shows that the directivity is increasing with the EBG patch width from 0.08 to 0.1 and then the middle part the directivity is almost constant. After that EBG patch width from 0.12, the directivity is decreasing in nature with the increasing EBG patch width.

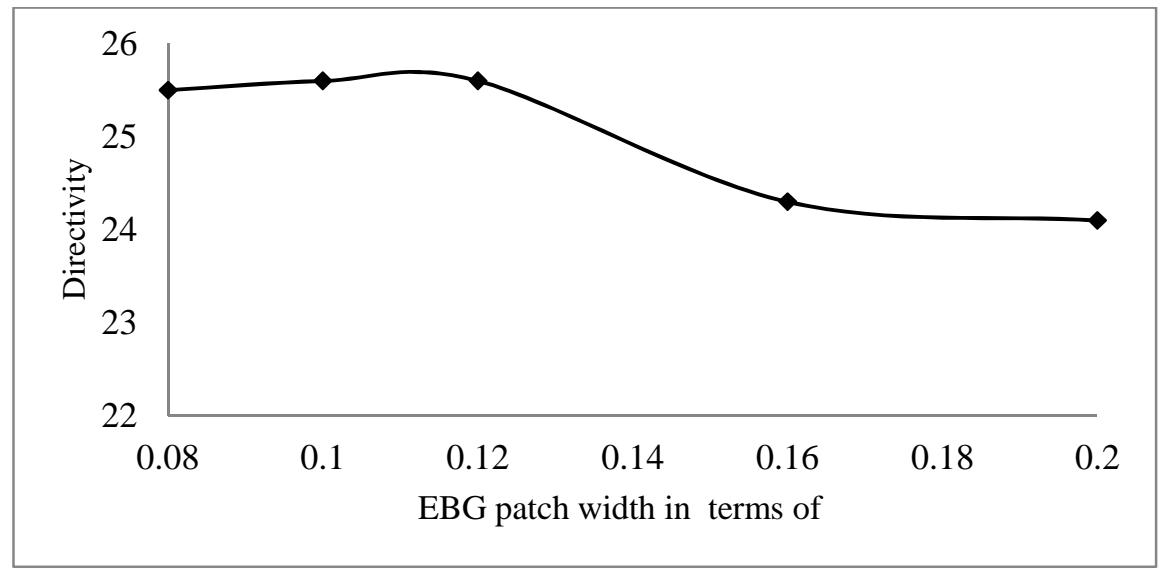

Fig 5.4 Directivity versus EBG patch width of the antenna on EBG substrate 
International Journal of Wireless \& Mobile Networks (IJWMN) Vol. 4, No. 5, October 2012

\section{Conclusion}

The most common application of patch antenna is in mobile communication. The goals of this paper is to design conventional patch antenna and the patch antenna integrated with EBG substrates of different EBG patch width, with same physical dimensions, that can operate at $10 \mathrm{GHz}$ and study the performance parameter of patch antenna when EBG structure added on it. From the simulated results, it is seen that the performance is better of a patch antenna that is designed on EBG substrates than the conventional patch antenna. It is also seen from the graphical analysis of performance parameter of patch antenna with EBG substrates, the most suitable performance is obtained at the range of EBG patch width is $0.08 \lambda_{12 \mathrm{GHZ}}$ to $0.12 \lambda_{12 \mathrm{GHZ}}$. In future, our targets are to real time implementation of the proposed antenna and also design another patch antenna with EBG substrates that can operate at higher frequency.

\section{REFERENCES}

[1] F.Benikhlef, N. Boukli-Hacene, "Influence of Side Effect of EBG Structures on the Far-Field Pattern of Patch Antennas”, IJCSI International Journal of Computer Science Issues, Vol. 9, Issue 1, No 3, January 2012.

[2] G.Gnanagurunathan and U.G.Udofia, "Performance analysis of the mushroom-like-EBG structure integrated with a microstrip patch antenna," Proceedings of IEEE Asia-Pacific Conference on Applied Electromagnetic (APACE), 2010.

[3] Peter KOVÁCS, Zbyněk RAIDA, Marta MARTÍNEZ-VÁZQUEZ "Parametric Study of Mushroom-like and Planar Periodic Structures in Terms of Simultaneous AMC and EBG Properties," Radio Engineering, Vol. 17, No. 4, December 2008.

[4] Zhixia Wei; Chenjiang Guo; Jun Ding; Nanjing Li; Zhibin Wei, "Parametric analysis of the EBG structure and its application for low profile wire antennas," 8th International Symposium on Antennas, Propagation and EM Theory( ISAPE), 2008.

[5] Jing Liang, and Hung-Yu David Yang, "Radiation Characteristics of a Microstrip Patch over an Electromagnetic Bandgap Surface," IEEE Transactions on Antennas and Propagation,Vol.55, pp1691-1697, June 2007.

[6] D. N. Elsheakh, H. A. Elsadek, E. A. Abdallah, H. Elhenawy, and M. F. Iskander,”Enhancement of Microstrip Monopole Antenna Bandwidth by Using EBG Structures," IEEE Antennas and Wireless Propagation Letters, vol. 8, pp 959-962, 2009.

[7] Fan Yang, Member, and Yahya Rahmat-Samii," Microstrip Antennas Integrated With Electromagnetic Band-Gap (EBG) Structures: A Low Mutual Coupling Design for Array Applications," IEEE Transactions on Antennas and Propagation, Vol. 51, No. 10, October 2003.

[8] Ram’on Gonzalo, Peter de Maagt, and Mario Sorolla, "Enhanced Patch-Antenna Performance by Suppressing Surface Waves Using Photonic-Bandgap Substrates," IEEE Transactions on Microwave Theory and Techniques, Vol. 47, pp. 2131-2138, November 1999.

[9] D. Qu, L. Shafai and A. Foroozesh, "Improving Microstrip Patch Antenna Performance Using EBG Substrates," IEE Proceedings Micro Antennas Propagation, Vol.153, pp.558-563, December 2006.

[10] Atsuya Ando, Kenichi Kagoshima, Akira Kondo, and Shuji Kubota, "Novel Microstrip Antenna With Rotatable Patch Fed by Coaxial Line for Personal Handy-Phone System Units," IEEE Transactions on Antennas and Propagation, Vol. 56, pp.2747-2751, August 2008.

[11] Mr. Pramod Kumar.M , Sravan kumar, Rajeev Jyoti , VSK Reddy, PNS Rao,"Novel Structural Design for Compact and Broadband Patch Antenna," IEEE International Workshop on Antenna Technology (iWAT), 2010.

[12] R. Chantalat, C. Menudier, M. Thevenot, T. Monediere, E. Arnaud, and P. Dumon, "Enhanced EBG Resonator Antenna as Feed of a Reflector Antenna in the Ka Band," IEEE Antennas and Wireless Propagation Letters, Vol. 7 pp.349-353, 2008. 
International Journal of Wireless \& Mobile Networks (IJWMN) Vol. 4, No. 5, October 2012

[13] Ioannis Papapolymerou, Rhonda Franklin Drayton, and Linda P. B. Katehi, "Micromachined patch Antennas," IEEE Transaction on Antenna Propagation, Vol.46, No.2, pp.275-283, 1998.

[14] Fan Yang, Yahya Rahmat Sami, "Electromagnetic band Gap Structures in Antenna Engineering," Cambridge University Press 2009.

[15] Constantine A. Balanis, "Antenna Theory Analysis and Design,” Third Edition, John Wiley \& Sons, 2005.

[16] D. M. Pozar, “Microwave Engineering,” Third Edition New York, Wiley, 2005.

[17] Fan Yang and Yahya Rahmat-Samii, Electromagnetic Band Gap Structures in antenna engineering, Cambridge University press, 2009.

[18] Fan Yang and Yahya Rahmat-Samii, ”Microstrip Antennas Integrated With Electromagnetic BandGap (EBG) Structures: A Low Mutual Coupling Design for Array Applications," IEEE Transactions on Antennas and Propagation, Vol. 51, No. 10, October 2003

\section{Authors}

Mst. Nargis Aktar received B.Sc. and M.Sc. in Electrical and Electronic Engineering from Rajshahi University of Engineering and Technology (RUET), Bangladesh in 2007 and 2010 respectively. She joined as a faculty member in Electrical and Electronic Engineering department of Ahsanullah University of Science \& Technology (AUST), Bangladesh, in 2007. In 2008 she joined as a faculty member in the department of Information and Communication Technology (ICT) of Mawlana Bhashani Science and Technology University (MBSTU), Bangladesh.

Muhammad Shahin Uddin received B.Sc. in Electrical and Electronic Engineering from Rajshahi University of Engineering and Technology (RUET), Bangladesh. He received his M.Sc. in the department of Electronics Engineering from Kookmin University, Korea. He joined as a faculty member in the department of Electrical and Electronic Engineering of Chittagong University of Engineering and Technology (CUET), Bangladesh in 2005. Since 2006 he is with the department of Information and Communication Technology (ICT) of Mawlana Bhashani Science and Technology University (MBSTU), Bangladesh.

Monir Morshed received his B.Sc. degree in Electrical and Electronic Engineering from Rajshahi University of Engineering and Technology (RUET), Bangladesh, in 2008. Then, he joined as a faculty member at the department of Information and Communication Technology of Mawlana Bhashani Science and Technology University (MBSTU), Bangladesh in 2008. His current research interests focus on computational electromagnatics, wireless communication, high frequency circuit design, antenna and propagation.

Dr. Md. Ruhul Amin is working as a Professor in Electrical and Electronic Engineering at the Islamic University of Technology, a subsidiary organ of OIC. He earned a Ph. D. in EEE from Niigata University, Japan, M. Sc. Engineering from Bangladesh University of Engineering and Technology and B. Sc Engineering from the University of Rajshahi. His research interests include generation and application of High Power Microwaves, Antenna theory and Signal Processing. He was the recipient of the Sir Thomas Ward memorial medal awarded by the Institution of
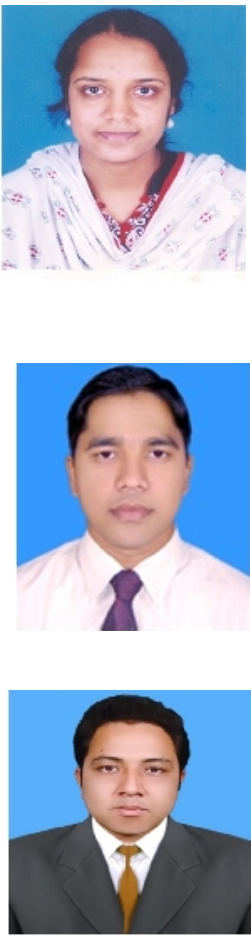
Engineers India. Professor Amin has published 66 journal and conference papers so far. He served as the Dean of the Faculty of Electrical and Computer Engineering at Rajshahi University of Engineering and 
International Journal of Wireless \& Mobile Networks (IJWMN) Vol. 4, No. 5, October 2012

Technology. Professor Amin is a Commonwealth Fellow and a Fellow of the Institution of Engineers, Bangladesh and a member of IEEE.

Dr. Md. Mortuza Ali was born in Bangladesh in 1957. He received the B. Sc. Engineering degree in Electrical and Electronic Engineering from Rajshahi University, Bangladesh in 1982, and the M. Sc. and Ph. D. degrees from Niigata University, Japan in 1989 and 1992, respectively. Since 2002 he has been employed by Rajshahi University of Engineering \& Technology, Bangladesh as Professor in the Department of Electrical and Electronic Engineering. His research interests include the high power microwave devices, antennas and wave propagation, and numerical analyses of various systems of linear/nonlinear equations.

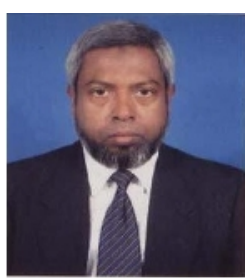

